\section{Post-traumatic chronic seronegative monarthritis responding to antitumor necrosis factor-alpha therapy}

\author{
Jamie Fairweather, MSc, MRCS, MRCEM, \\ Ali S. Jawad, MSc, FRCP.
}

$\mathrm{T}$ rauma has been reported to trigger inflammatory arthritis. ${ }^{1,2}$ Studies have shown that the relationship between trauma and the development of psoriatic arthritis is stronger than that between trauma and rheumatoid arthritis. ${ }^{2,3}$

A 31-year-old woman was referred to the rheumatology department due to persistent effusion of the left knee. She was a keen long-distance runner (several miles a week) and 5 years earlier, she had received treatment elsewhere because she developed pain and swelling of her left knee, exacerbated by knee flexion. She was otherwise fit and well with no other past medical history of note. Magnetic resonance imaging (MRI) of the knee demonstrated high signal in the hamstring tendon sheath, pes anserinus bursa and some thickening of the anterior cruciate ligament, but the fibres were intact. There was also a small effusion. She was diagnosed with a pes anserine bursitis and hamstring tendinopathy and underwent ultrasoundguided steroid injection, but this did not produce any sustainable benefit. She was unable to run but was able to walk unaided. Two years later, she saw another orthopedic surgeon. He excised a ganglion on the medial aspect of the same knee and the knee was drained and injected with corticosteroids. Within a few weeks the pain and swelling recurred. Another MRI scan showed joint effusion. Arthroscopy was performed and a synovial biopsy showed chronic inflammatory synovitis. Cultures were negative including acid fast bacilli. She was treated with aspiration and intra-articular corticosteroid injection (repeated several times), non-steroidal anti-inflammatories, sulfasalazine and hydroxychloroquine, but without any improvement. She declined methotrexate as she was trying to conceive.

At presentation in our department, there was no other joint involvement and no history of mouth ulcers, back pain, psoriasis, nail dystrophy, Raynaud's phenomenon, ocular pathology, bowel or bladder disturbance. There was no family history of psoriasis. Physical examination showed a moderate effusion of the left knee. There were no other physical signs. Aspiration of the synovial fluid showed intense neutrophilic infiltration but no crystals were seen and cultures were negative. Her erythrocyte sedimentation rate was 34 $\mathrm{mm} /$ hour. C-reactive protein was $<5 \mathrm{~g} / \mathrm{dl}$. Antinuclear antibodies were negative. Antibodies to the extractable nuclear antigens, HLA-B27, rheumatoid factor and a cyclic citrullinated peptide antibody test were negative. We reviewed the original synovial biopsy and it showed congestion and edema of the synovium with moderately severe infiltration by lymphocytes, plasma cells and histiocytes with deposition of fibrinous material over the synovial service but no evidence of vasculitis, crystal, granuloma or lymphoid follicle formation. (Figure 1).

A diagnosis of post traumatic chronic monarthritis of the left knee was made and antitumor necrosis factoralpha (TNF $\alpha)$ was considered. After 3 fortnightly injections of adalimumab $40 \mathrm{mg}$ subcutaneously, the patient's knee had improved dramatically and the large effusion, which had been persistent for the previous 5 years, resolved. She completed 6 months of treatment with adalimumab. She experienced no recurrence of her symptoms for 27 months after stopping the adalimumab. She was running again, but mainly on grass and shorter distances. A spontaneous recurrence of the effusion of the left knee occurred when she was 7 weeks pregnant. The knee was aspirated and injected with corticosteroids with good initial response. Three weeks later she had a spontaneous abortion and within a fortnight the effusion of the knee recurred. Again, the knee was aspirated and injected with corticosteroids but within 3 weeks the effusion recurred. She was treated with adalimumab $40 \mathrm{mg}$ subcutaneously every

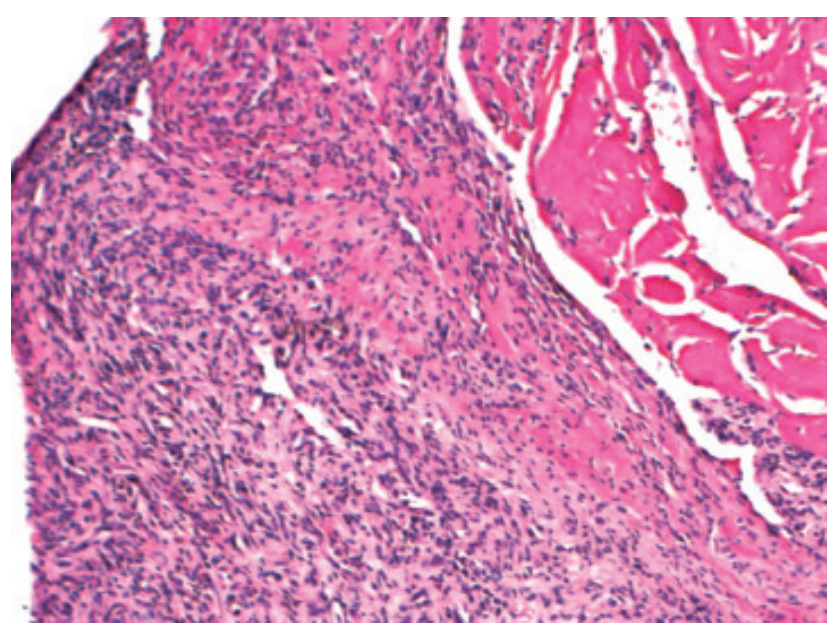

Figure 1 - Synovial biopsy (H\&E x200) showing congestion and oedema of the synovium with moderately severe infiltration by lymphocytes, plasma cells and histiocytes with deposition of fibrinous material over the synovial service but no evidence of vasculitis, crystal, granuloma or lymphoid follicle formation. 
other week with rapid resolution of the effusion. Six months later, she remains symptom free. Four years later, the patient remains symptom free on monthly adalimumab.

Trauma can trigger inflammatory and crystal arthritis. ${ }^{2}$ Our patient was a keen long-distance runner and ran several miles a week on hard surfaces, roads and pavements. There was no preceding specific injury and the MRI imaging failed to show any abnormality apart from the effusion. The history, physical examination and investigations did not reveal any specific underlying cause. The synovial biopsy confirmed the inflammatory nature of the synovitis. We assumed that the repeated trauma of running must have been the triggering factor. A diagnosis of post-traumatic chronic seronegative monarthritis was made. The majority of cases of post traumatic synovitis settle spontaneously. However, in our patient's case the synovitis persisted for more than 4 years. The differential diagnosis also includes the possibility of psoriatic arthritis preceding the development of psoriasis with the arthritis being precipitated by the trauma. She failed to respond to repeated aspiration and intraarticular corticosteroid injections, non-steroidal antiinflammatories, sulfasalazine and hydroxychloroquine. She declined methotrexate. The response to adalimumab was dramatic with no recurrence for 4 years and the dose of adalimumab was reduced to monthly injections. Because our patient did not satisfy the United Kingdom national guidelines for the use of anti TNF $\alpha$, we had to get special permission from the health authorities to permit its use in this case. ${ }^{4}$

We conclude that a trial of adalimumab for a short period may be justifiable in cases of post-traumatic chronic seronegative synovitis unresponsive to standard therapy.

Received 29th January 2018. Accepted 7th March 2018.

From the Department of Emergency Medicine (Fairweather), and the Department of Rheumatology (Jawad), The Royal London Hospital, London, United Kingdom. Address correspondence and reprints request to: Prof. Ali S. Jawad, Department of Rheumatology, The Royal London Hospital, London, United Kingdom.E-mail:ali.jawad@bartshealth.nhs.uk

ORCID ID: orcid.org/0000-0002-0434-7488

\section{References}

1. Currey J, Therkildsen LH, Bywaters EG. Monarticular rheumatoid-like arthritis of seven years' duration following fracture of the radial head. Ann Rheum Dis 1986; 45: 783-785.

2. Olivieri I, Padula A, D'Angelo S, Scarpa R. Role of Trauma in Psoriatic Arthritis. J Rheumatol 2008; 35: 2085-2087.

3. Pattison E, Harrison BJ, Griffiths CE, Silman AJ, Bruce IN. Environmental risk factors for the development of psoriatic arthritis: results from a case-control study. Ann Rheum Dis 2008; 67: 672-676.

4. The National Institute for Health and Care Excellence (NICE). Adalimumab, etanercept, infliximab, certolizumab pegol, golimumab, tocilizumab and abatacept for rheumatoid arthritis not previously treated with DMARDs or after conventional DMARDs only have failed. [Update 2016 January 26; Accessed 2018 February 27). Available from URL: https://www.nice. org.uk/guidance/ta375 